\title{
Interpretation of Contradictory Images by Means of Systems of Linear Inequalities
}

\author{
V. D. Mazurov ${ }^{1,2}$ and A. I. Smirnov ${ }^{1}$ \\ Received February 3, 2012
}

\begin{abstract}
We consider the problem of interpretation of three-dimensional images from their flat projections up to the set of visible faces. For projections of convex polyhedra, we present an interpretation algorithm based on maximal feasible subsystems of a certain infeasible system of linear inequalities modeling the visibility requirement for faces. A number of model examples are given; in particular, the algorithm is applied to the interpretation of the Necker cube.
\end{abstract}

Keywords: polyhedron, face, interpretation, linear inequalities.

DOI: $10.1134 / \mathrm{S} 0081543813090101$

\section{INTRODUCTION}

In S.N. Chernikov and his pupils' papers, a universal approach to the investigation of systems of linear inequalities was developed. The approach included the method of boundary solutions, the method of fundamental convolution, and other basic methods, which are applicable not only to feasible but also to infeasible systems of linear inequalities. Since the techniques from the theory of linear inequalities were widely used in the simulation of all kinds of systems, these methods were applied in various areas of research. As will be shown below, one of such new application domains is the analysis of flat images of three-dimensional objects.

The issues of automatic image processing have recently become especially important. The problems of developing applied intellectual and robotic systems and related problems of understanding the mechanisms of human vision, as well as the problem of image interpretation in computer tomography, initiated the growth of investigations in the area of image processing, which produced important results in the last ten years [1]. In particular, there was a significant progress in the development of solution methods for problems of recovering three-dimensional objects from twodimensional digital images. Here, we apply the method of maximal feasible subsystems (MFSs) for recovering the three-dimensional structure of a polyhedron from its known orthogonal projections.

The classical statement of this problem assumes the presence of several aspects of the same object, which makes it possible to develop algorithms based on well-tested projective methods (see review [1]) such as for example, the algorithm of recovery from a stereo pair (the standard and wide-base stereo). However, at present, researchers become more interested in the recovery of

\footnotetext{
${ }^{1}$ Institute of Mathematics and Mechanics, Ural Branch of the Russian Academy of Sciences, ul. S. Kovalevskoi 16, Yekaterinburg, 620990 Russia

emails: vladimir.mazurov@usu.ru, sm@urep.ru

${ }^{2}$ Graduate School of Economics and Management, Ural Federal University, ul. Mira 19, Yekaterinburg, 620002 Russia
} 

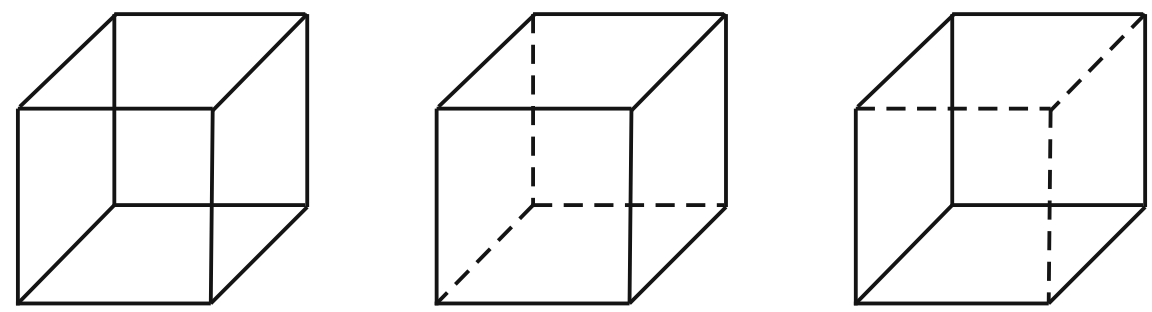

Fig. 1. The Necker cube and its possible spatial interpretations.

three-dimensional scenes from one image $[2,3]$. In this connection, let us mention the Make3D project of Cornell University [4], which was started at Stanford University. The aim of the project is to solve the problem of recovering a three-dimensional model of a scene from only one photograph; this problem has not yet become typical. The project has shown that a considerable amount of information is contained in the so-called monocular cues of an image, which often were ignored before. In the understanding of the mechanisms of forming visible patterns, it is especially interesting to analyze ambiguous images, which admit two or more interpretations (the so-called transformable or reversible images).

Here, we consider a variant of this problem consisting in the interpretation of flat projections of three-dimensional objects, which are transparent polyhedra given by their skeletons. This problem is important not only for applications but also from the point of view of understanding the mechanisms of image perception and interpretation by the human brain. It is especially interesting to consider the human perception of so-called dual and contradictory images; this problem has been heavily studied in recent years by philosophers, psychologists, and mathematicians [5].

One of the simplest and most studied images of this kind is the Necker cube. This is an outline image corresponding to a parallel projection of vertices and edges of a cube to a plane. This image and its two possible space interpretations are given in Fig. 1.

If this image is observed long enough, it seems to 'reverse' spontaneously: one three-dimensional projections is replaced by another. Apparently, the two interpretations have equal rights in this case, and the brain 'tries' each of these conjectures in turns, having no grounds to finally adopt any of them. This happens due to the complete absence of contextual information, which usually helps to make the decision in the recognition of visual images (since there is no perspective, the faces have equal size). Other known examples of similar type are the outline of a half-open book (Mach's figure) and the Schröder staircase [6].

Patterns of perception of such ambiguous images have been analyzed in detail, in particular, by Caglioti [7], who showed that these patterns are in many respect similar to self-organization phenomena in dissipative structures beyond an unstable critical state. The peculiarity of these processes is the dynamic alternation (inversion) of two stationary states. The Necker cube represents the class of 'wireframe models' [8], which contain only vertices and edges of an object.

The aim of this paper is to demonstrate a new approach to solving problems of three-dimensional interpretation of flat images based on the search for maximal (with respect to inclusion) feasible subsystems of a certain infeasible system of linear inequalities. The class of three-dimensional objects under consideration is the class of convex polyhedra, and the inequalities of the mentioned infeasible system of constraints correspond to the visibility requirement for the faces of a polyhedron.

Since any real (plain) image can be approximated by an image consisting of points only, we understand an image as a nonempty set of points on a plane as in [1]. 


\section{PROBLEM STATEMENT}

Since the proposed methods use mathematical techniques from the theory of infeasible systems of inequalities [9-13] and are not restricted to the case of three-dimensional scenes, we give a general statement of the problem $[14,15]$.

Assume that $L_{1}$ and $L_{2}$ are real linear spaces, $L=L_{1} \times L_{2}$ is their Cartesian product, and the projection $P$ of a convex polyhedron $Q \subset L$ to the subspace $L_{1}$ is known:

$$
P=\bigcup_{i=1}^{k} M_{P}^{i}=\pi_{L_{1}} \bigcup_{i=1}^{k} M_{Q}^{i} .
$$

Here, $\pi_{L_{1}}$ is the operator of projection to $L_{1}, M_{Q}^{i}(i=1, \ldots, k)$ is the set of faces of the original polyhedron of dimension $i-1$, and $M_{P}^{i}$ is the set of their projections. Thus, $M=M_{P}^{1}$ is the set of point projections of vertices of the polyhedron, $M_{P}^{2}$ is the set of projections of edges, and so on. In what follows, we assume that the set $M$ is ordered. It is required to reconstruct $Q$ in the form

$$
Q=\left\{q=\left[p, y_{p}\right] \in L: p \in P\right\},
$$

where $p \in L_{1}, y_{p} \in L_{2}$, and $y=\left[y_{p} \in L_{2}: p \in P\right]$ is the required vector.

The proposed approach consists in the following. Let $q_{i}=\left[p_{i}, y_{i}\right](i=1, \ldots, t)$ be vertices of $Q$ that define its face $q_{1} q_{2} \ldots q_{t}$. Assume that a predicate $V$ is defined in some way on the set of tuples $\left[q_{1} q_{2} \ldots q_{t}\right]$ (in accordance with projections). A face $q_{1} q_{2} \ldots q_{t}=\operatorname{co}\left\{q_{1}, q_{2}, \ldots, q_{t}\right\}$, which corresponds to the tuple $\left[q_{1} q_{2} \ldots q_{t}\right]$, is called visible if $V\left(q_{1} q_{2} \ldots q_{t}\right)=1$. Then, the collections of visible faces of $Q$ correspond to MFSs of the system

$$
V\left(q_{1} q_{2} \ldots q_{t}\right)=1 \quad\left(\forall p_{1} p_{2} \ldots p_{t} \subset P\right) .
$$

Let us consider in detail the problem of recovering a three-dimensional polyhedron from its flat projection. Suppose that there is a three-dimensional polyhedron $Q$ defined by the sets of its vertices $M_{Q}$, edges $M_{Q}^{2}$, and faces $M_{Q}^{3}$. Let the projection $P$ of this polyhedron to a plane given by its normal vector $\vec{n}$ be known. It is required to recover the original polyhedron from the projection up to some equivalence relation, which is defined below. The recovered polyhedron will be called an interpretation of the projection. The same terminology will be used for the constituent parts: vertices, edges, and faces. Let the projections of vertices, edges, and faces of the original polyhedron $Q$ form the sets $M_{P}, M_{P}^{2}$, and $M_{P}^{3}$, respectively.

Let us introduce a rectangular coordinate system $x_{1}, x_{2}$ on the projection plane. The third coordinate $y$ will be directed along the vector $\vec{n}$. Thus, each vertex $q=\left(x_{1}, x_{2}, y\right)$ of the polyhedron $Q$ corresponds to some point $p=\left(x_{1}, x_{2}\right)$ of the figure $P$. We introduce an equivalence relation on the set of inverse images of $P$ and assume the polyhedron recovery problem to be solved if representatives of all equivalence classes are found.

Introduce on the set of faces $M_{Q}^{3}$ of $Q$ the following order relation.

Definition 1. A face $\Gamma_{1}$ precedes a face $\Gamma_{2}$ if there exists a point $x=\left(x_{1}, x_{2}\right)$ that belongs to the interior of the intersection $\gamma_{1} \cap \gamma_{2}$ of the projections $\gamma_{1}$ and $\gamma_{2}$ of these faces and has the inverse images $z_{1}=\left(x_{1}, x_{2}, y_{1}\right)$ and $z_{2}=\left(x_{1}, x_{2}, y_{2}\right)$ on the faces $\Gamma_{1}$ and $\Gamma_{2}$, respectively, and $y_{1}<y_{2}$.

Since the choice of the point $x$ in this definition is ambiguous, it is necessary to show that the definition is correct, i.e., that it is independent of the choice of this point. Assume that the following conditions hold: 
(A1) $Q$ is a convex polyhedron,

(A2) $Q$ is a bounded polyhedron,

(A3) $Q$ is a solid polyhedron.

Lemma 1. Let $x=\left(x_{1}, x_{2}\right)$ and $x^{\prime}=\left(x_{1}^{\prime}, x_{2}^{\prime}\right)$ be any two points from the interior of the intersection $\gamma_{1} \cap \gamma_{2}$ of the projections $\gamma_{1}$ and $\gamma_{2}$ of faces $\Gamma_{1}$ and $\Gamma_{2}$, respectively, with inverse images $z_{1}=\left(x_{1}, x_{2}, y_{1}\right), z_{1}^{\prime}=\left(x_{1}^{\prime}, x_{2}^{\prime}, y_{1}^{\prime}\right)$ and $z_{2}=\left(x_{1}, x_{2}, y_{2}\right), z_{2}^{\prime}=\left(x_{1}^{\prime}, x_{2}^{\prime}, y_{2}^{\prime}\right)$ on the faces $\Gamma_{1}$ and $\Gamma_{2}$, respectively. Then, the inequalities $y_{1}<y_{2}$ and $y_{1}^{\prime}<y_{2}^{\prime}$ hold or do not hold simultaneously.

Proof. The proof is by contradiction. Assume that there exists a pair of points $x=\left(x_{1}, x_{2}\right)$ and $x^{\prime}=\left(x_{1}^{\prime}, x_{2}^{\prime}\right)$ that satisfy the condition of the lemma. Assume, by contradiction, that $y_{1}<y_{2}$ and $y_{1}^{\prime}>y_{2}^{\prime}$. Consider the intervals $\left[z_{1}, z_{1}^{\prime}\right]$ and $\left[z_{2}, z_{2}^{\prime}\right]$. Since $x$ and $x^{\prime}$ are interior points of the projections $\gamma_{1}$ and $\gamma_{2}$ of the faces $\Gamma_{1}$ and $\Gamma_{2}$, respectively, it follows that the points $z_{1}, z_{1}^{\prime}, z_{2}, z_{2}^{\prime}$ are interior points of their faces. Since the points $z_{1}, z_{2}$ and $z_{1}^{\prime}, z_{2}^{\prime}$ lie on parallel straight lines, the specified intervals lie in the plane defined by these straight lines, and all their points are interior points of the faces $\Gamma_{1}$ and $\Gamma_{2}$, respectively. Then, however, these intervals intersect at some point $\tilde{z}$, which belongs to the faces $\Gamma_{1}$ and $\Gamma_{2}$ simultaneously. Consequently, $\tilde{z} \in \Gamma_{1} \cap \Gamma_{2}$ and $\Gamma_{1} \cap \Gamma_{2} \neq \varnothing$. Thus, the faces $\Gamma_{1}$ and $\Gamma_{2}$ intersect, and $\tilde{z}$ is an interior point of each of the faces, which is impossible. The contradiction proves the lemma.

Lemma 2. Let conditions (A1)-(A3) be satisfied. Then, for any face $\Gamma$ of the polyhedron $Q$, there exists exactly one other face comparable with $\Gamma$ with respect to the introduced order relation.

Proof. Let $x=\left(x_{1}, x_{2}\right)$ be some interior point of the projection $\gamma$ of some face $\Gamma$ of the polyhedron $Q$, and let $z=\left(x_{1}, x_{2}, y\right) \in \Gamma$. Consider the straight line $l$ passing through the point $x$ parallel to the $O y$ axis (i.e., orthogonal to the plane of the projection $P$ ). Let us show that this straight line intersects at least one more face of $Q$.

By condition (A3), there exists an interior point $z_{1}$ of the polyhedron $Q$ not lying on the face $\Gamma$. Let $z_{1}^{\prime}$ be the point of intersection of the face $\Gamma$ with the perpendicular drawn from $z_{1}$ to the projection plane. By condition (A1), the interval $\left[z_{1}, z\right]$ completely consists of points of the polyhedron $Q$; hence, we can assume that $z_{1}^{\prime}$ is an interior point of $\Gamma$. Let $z_{2}$ be a point on the extension of the interval $\left[z_{1}^{\prime}, z\right]$ that also belongs (together with the point $z$ ) to the face $\Gamma$ and, in addition, lies inside the interval $\left[z_{1}^{\prime}, z_{2}\right]$. Then, the interval $\left[z_{1}, z_{2}\right]$ intersects the straight line $l$ at some point $z_{3} \in Q$. Thus, there is one more point $z_{3} \neq z$ of the polyhedron $Q$ on the straight line $l$; consequently, the interval $\left[z, z_{3}\right]$ completely consists of points of the polyhedron $Q$. As follows from condition (A2), the ray that continues the interval $\left[z, z_{3}\right]$ contains a point not belonging to $Q$ and, consequently, a point $z^{\prime} \in l$ lying on the boundary of $Q$, i.e., on some of its faces $\Gamma^{\prime}$.

Thus, it is shown that the straight line $l$ intersects $Q$ at least at two points $z$ and $z^{\prime}$. Let us show that there are no other boundary points of $Q$ on this line.

Assume the existence of a point $z_{0}$ such that $z_{0} \neq z, z_{0} \neq z^{\prime}$, and $z_{0} \in l \cap Q$. Let these points lie on $l$, for example, in the order $z, z^{\prime}, z_{0}$. Then, the point $z^{\prime}$, on the one hand, is a boundary point of $Q$ because $z^{\prime} \in \Gamma^{\prime}$ and, on the other hand, by condition (A1), is an interior point of $Q$ because $z^{\prime} \in\left(z, z_{0}\right)$. This contradiction shows that the line $l$ intersects exactly two faces of $Q$. According to the above definition, this means that there are exactly two comparable faces $\Gamma$ and $\Gamma^{\prime}$.

The lemma is proved.

In accordance with the natural interpretation, elements of $M_{Q}^{3}$ that are minimal with respect to the introduced order will be called visible faces. Polyhedra in which faces with the same projection are visible or invisible simultaneously will be called equivalent. Thus, the problem of interpreting 
a flat projection of a polyhedron can be refined as follows: reconstruct the original polyhedron up to the collection of visible faces.

Note that this problem is equivalent to the problem of finding minimal (with respect to inclusion) covers of the convex hull co $M_{P}$ of projections of vertices of $Q$ by projections of its faces. This circumstance, on the one hand, provides a possible approach to the solution of the original problem and, on the other hand, can serve as a solution criterion for a found collection of faces.

Remark. A collection of visible faces, evidently, depends on the viewing direction, i.e., on the direction of the $O y$ axis. If the direction of this axis is changed for the opposite, then, obviously, maximal faces become minimal and, vice versa, minimal faces become maximal.

Let us formulate the main result.

Theorem 1. The problem of interpreting a flat projection of a polyhedron under conditions (A1)-(A3) has exactly two solutions: the first is the collection of minimal faces of $Q$, and the second is the collection of its maximal faces.

Proof. The proof of this theorem follows from the proved lemmas and from the remark.

\section{INTERPRETATION OF VERTICES, EDGES, AND FACES}

Note that edges belonging to the boundary of the projection co $M_{P}$ of the polyhedron are visible in all interpretations. Further, the visibility of faces of larger dimension (edges and faces in the three-dimensional case) implies the visibility of their subsets that are faces of smaller dimension (vertices and edges, respectively).

Before solving the problem, it is useful to verify the fulfillment of some simple conditions that follow from our requirements: the Euler relation $\left|M_{P}\right|-\left|M_{P}^{2}\right|+\left|M_{P}^{3}\right|=2$ and the condition that there are at least three edges emanating from each vertex.

Let us pass to the interpretation of vertices. Let $p \in M_{P}$. If this point lies on the boundary of the projection of the polyhedron, then this vertex is visible in any interpretation. Now, let the point $p$ lie inside the projection of the polyhedron. Then, there exists a face $p_{i_{1}} p_{i_{2}} \ldots p_{i_{k}}$ containing this point. We find the expansion

$$
p=\sum_{j=1}^{k} \alpha_{j} p_{i_{j}}, \quad \alpha_{j} \geq 0 \quad(\forall j \in \overline{1, k}), \quad \sum_{j=1}^{k} \alpha_{j}=1 .
$$

If $p_{i_{j}}=\left(x_{1}^{i_{j}}, x_{2}^{i_{j}}\right), q_{i_{j}}=\left(x_{1}^{i_{j}}, x_{2}^{i_{j}}, y_{j}\right), p=\left(x_{1}, x_{2}\right)$, and $q=\left(x_{1}, x_{2}, y\right)$, then the vertex $q$ is considered to be visible if $y<\sum_{j=1}^{k} \alpha_{j} y_{j}$.

Let us now consider the interpretation of edges. As noted earlier, the visibility of the vertices of an edge (if at least one of them does not belong to the boundary of $\operatorname{co} M_{p}$ ) is sufficient for the visibility of the edge. However, the method of interpretation of projections based on the interpretation of vertices does not guarantee that the above conditions hold for the obtained polyhedron; the class of interpretations is too wide in this case and contains various 'elaborate' figures. Therefore, we will use the following refinement of the method of interpretation of vertices.

Consider an edge $p_{1} p_{2} \in M_{P}^{2}$. Let $p_{1}=\left(x_{1}^{1}, x_{2}^{1}\right), p_{2}=\left(x_{1}^{2}, x_{2}^{2}\right), q_{1}=\left(x_{1}^{1}, x_{2}^{1}, y_{1}\right), q_{2}=\left(x_{1}^{2}, x_{2}^{2}, y_{2}\right)$, $p_{j}^{i}=\left(x_{1}^{i, j}, x_{2}^{i, j}\right)$, and $q_{j}^{i}=\left(x_{1}^{i, j}, x_{2}^{i, j}, y_{i j}\right)\left(i \in \overline{1, r}, j \in \overline{1, k_{i}}\right)$. Let us find all the faces $\Gamma_{i}=p_{1}^{i} \ldots p_{k_{i}}^{i}$ $(i \in \overline{1, r})$ that intersect the edge $p_{1} p_{2}$. We construct the expansions

$$
p_{1}=\sum_{s=1}^{k_{i}} \alpha_{1 s}^{i} p_{s}^{i}, \quad p_{2}=\sum_{s=1}^{k_{i}} \alpha_{2 s}^{i} p_{s}^{i}, \quad \sum_{s=1}^{k_{i}} \alpha_{t s}^{i}=1 \quad(t=1,2) .
$$


Further, we consider the edge $q_{1} q_{2}$ to be visible if and only if the following inequalities are satisfied:

$$
y_{1}<\sum_{s=1}^{k_{i}} \alpha_{1 s}^{i} y_{i s}, \quad y_{2}<\sum_{s=1}^{k_{i}} \alpha_{2 s}^{i} y_{i s} \quad(i \in \overline{1, r}) .
$$

We obtain similar systems for all edges from $M_{P}^{2}$ and combine them:

$$
\left.\begin{array}{c}
A_{1} y<0 \\
\cdots \\
A_{n} y<0
\end{array}\right\}
$$

Here, $n=\left|M_{P}^{2}\right|$ and $y$ is the vector composed of the found values $y_{1}, y_{2}, y_{i s}$ (its dimension is $\left|M_{P}\right|$ ).

We should also take into account the absence of 'bends' of faces with more than three vertices. This gives a (possibly, empty) system of equalities of the form $B y=0$, which allows us to lower the dimension of system (1). As a result, we obtain the system of inequalities

$$
\left.\begin{array}{c}
\tilde{A}_{1} \tilde{y}<0 \\
\tilde{A}_{n} \tilde{y}<0
\end{array}\right\}
$$

where the vector $\tilde{y}$ contains only a part of variables of the vector $y$.

Further, we find all MFSs of system (2) and, among them, select subsystems that are sufficient for the interpretation of edges, i.e., are composed of some matrices $\tilde{A}_{i}$ only. Thus, this algorithm completely solves the formulated interpretation problem.

Consider some model examples.

\section{EXAMPLES OF THE OPERATION OF THE ALGORITHM}

Example 1. Consider the following projection (Fig. 2): $M_{P}=\left\{p_{1}, p_{2}, p_{3}, p_{4}\right\}, p_{1}=(-1,0)$, $p_{2}=(0,-1), p_{3}=(1,2), p_{4}=(2,0)$, and $M_{P}^{1}=\left\{p_{1} p_{2}, p_{1} p_{3}, p_{1} p_{4}, p_{2} p_{3}, p_{2} p_{4}, p_{3} p_{4}\right\}$.

The edges $q_{1} q_{4}$ and $q_{2} q_{3}$ can be invisible.

(1) The edge $q_{1} q_{4}$. If $q_{1}$ 'screened' by the extension of the face $q_{2} q_{3} q_{4}$, then

(a1) $5 y_{1}<6 y_{2}+3 y_{3}-4 y$.

If $q_{4}$ 'screened' by the extension of the face $q_{1} q_{2} q_{3}$, then

(a2) $4 y_{4}<-5 y_{1}+6 y_{2}+3 y_{3}$.

(2) The edge $q_{2} q_{3}$. If $q_{2}$ is 'screened' by the extension of the face $q_{1} q_{3} q_{4}$, then

(a3) $6 y_{2}<5 y_{1}-3 y_{3}+4 y_{4}$.

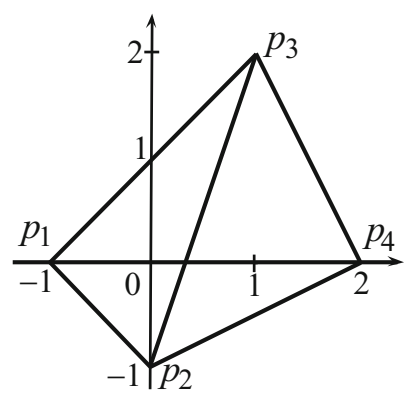

Fig. 2.

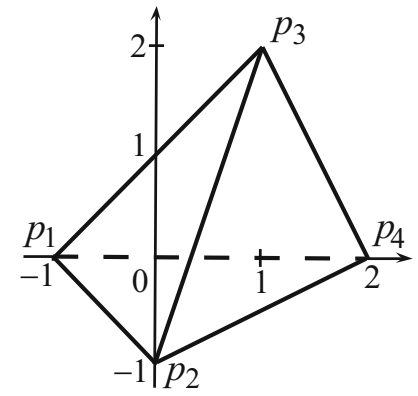

Fig. 3.

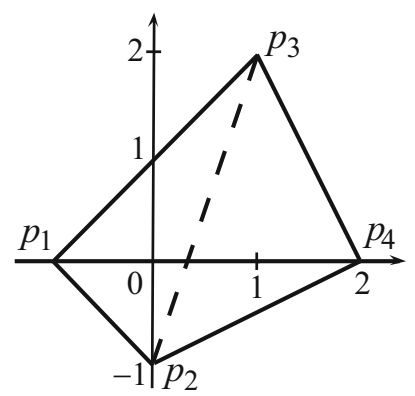

Fig. 4. 
Writing $h(y)=5 y_{1}-6 y_{2}-3 y_{3}+4 y_{4}$, we obtain the system of inequalities

$$
\begin{aligned}
& \text { (a1) } \quad h(y)<0 \text {, } \\
& \text { (a2) } \quad h(y)<0 \text {, } \\
& \text { (a3) }-h(y)<0 \text {, } \\
& \text { (a4) }-h(y)<0 \text {, }
\end{aligned}
$$

which has two MFSs. The first MFS, which contains the first two inequalities, corresponds to the visibility of the edge $q_{2} q_{3}$ (Fig. 3), whereas the second MFS, which contains the last two inequalities, corresponds to the visibility of the edge $q_{1} q_{4}$ (Fig. 4).

Example 2. Let $M_{P}=\left\{p_{1}, p_{2}, p_{3}, p_{4}, p_{5}\right\}, p_{1}=(-3,0), p_{2}=(-1,-1), p_{3}=(0,4), p_{4}=(1,1)$, $p_{5}=(2,0)$, and $M_{P}^{1}=\left\{p_{1} p_{2}, p_{1} p_{3}, p_{1} p_{4}, p_{2} p_{3}, p_{2} p_{5}, p_{3} p_{4}, p_{3} p_{5}, p_{4} p_{5}\right\}$ (see Fig. 5).

The edges $q_{1} q_{4}, q_{2} q_{3}, q_{3} q_{4}$, and $q_{4} q_{5}$ can be invisible.

(1) The edge $q_{1} q_{4}$ :

(b1) $q_{1} \in q_{2} q_{3} q_{5} \Rightarrow 14 y_{1}<20 y_{2}+5 y_{3}-11 y_{5}$

(b2) $q_{4} \in q_{2} q_{3} q_{5} \Rightarrow 7 y_{4}<y_{2}+2 y_{3}+4 y_{5}$,

(b3) $q_{4} \in q_{1} q_{2} q_{3} \Rightarrow 11 y_{4}<-8 y_{1}+13 y_{2}+6 y_{3}$.

(2) The edge $q_{2} q_{3}$ :

(b4) $q_{2} \in q_{1} q_{3} q_{4} \Rightarrow 13 y_{2}<8 y_{1}-6 y_{3}+11 y_{4}$,

(b5) $q_{3} \in q_{1} q_{2} q_{4} \Rightarrow 6 y_{3}<8 y_{1}-13 y_{2}+11 y_{4}$.

(3) The edge $q_{3} q_{4}$ :

(b6) $q_{4} \in q_{2} q_{3} q_{5} \Rightarrow 7 y_{4}<y_{2}+2 y_{3}+4 y_{5}$.

(4) The edge $q_{4} q_{5}$ :

(b7) $q_{4} \in q_{2} q_{3} q_{5} \Rightarrow 7 y_{4}<y_{2}+2 y_{3}+4 y_{5}$.

The equation corresponding to the requirement $q_{5} \in q_{1} q_{2} q_{4}$ has the form

(b8) $4 y_{1}-5 y_{2}-5 y_{4}+6 y_{5}=0$.

Using this equation and setting $h(y)=14 y_{1}-20 y_{2}-5 y_{3}+11 y_{5}$, we get the system of inequalities

$$
\begin{aligned}
& \text { (b1) } \quad h(y)<0 \text {, } \\
& \text { (b2) } \quad h(y)<0 \text {, } \\
& \text { (b3) } \quad h(y)<0 \text {, } \\
& \text { (b4) }-h(y)<0 \text {, } \\
& \text { (b5) }-h(y)<0 \text {, } \\
& \text { (b6) } \quad h(y)<0 \text {, } \\
& \text { (b7) } \quad h(y)<0 \text {. }
\end{aligned}
$$

This system has two MFSs: the first of them contains the fourth and fifth inequalities, whereas the second contains the remaining inequalities. Therefore, we obtain two interpretations: in the first, the visible edges are $q_{1} q_{4}, q_{3} q_{4}$, and $q_{4} q_{5}$ (Fig. 6); in the second, the visible edge is $q_{2} q_{3}$ (Fig. 7).

Example 3. The Necker cube [7] (Fig. 8).

Let $M_{P}=\left\{p_{1}, p_{2}, \ldots, p_{8}\right\}$, where $p_{1}=(0,0), p_{2}=(0,1), p_{3}=(0.25,0.5), p_{4}=(0.25,1.5)$, $p_{5}=(1,0), p_{6}=(1,1), p_{7}=(1.25,0.5)$, and $p_{8}=(1.25,1.5)$. The set of edges is $M_{P}^{2}=$ $\left\{p_{1} p_{2}, p_{1} p_{3}, p_{1} p_{5}, p_{2} p_{4}, p_{2} p_{6}, p_{3} p_{4}, p_{3} p_{7}, p_{4} p_{8}, p_{5} p_{6}, p_{5} p_{7}, p_{6} p_{8}, p_{7} p_{8}\right\}$, where $\left|M_{P}^{2}\right|=12$ and each point $p_{i}=\left(x_{1}^{i}, x_{2}^{i}\right)$ corresponds to a vertex $q_{i}=\left(x_{1}^{i}, x_{2}^{i}, y_{i}\right)$ of a polyhedron in the three-dimensional space. The edges $p_{1} p_{2}, p_{2} p_{4}, p_{4} p_{8}, p_{7} p_{8}, p_{5} p_{7}$, and $p_{1} p_{5}$ are always visible (in any interpretation). 


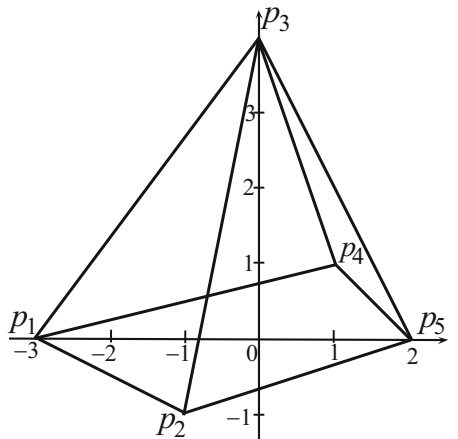

Fig. 5.

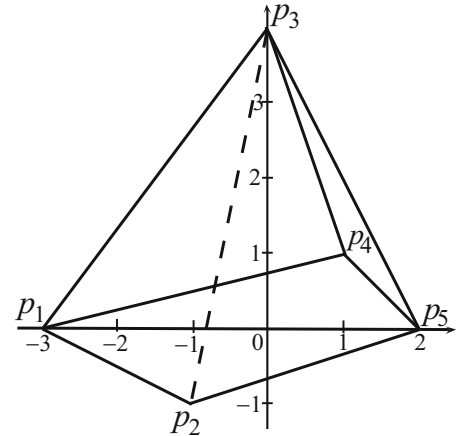

Fig. 6.

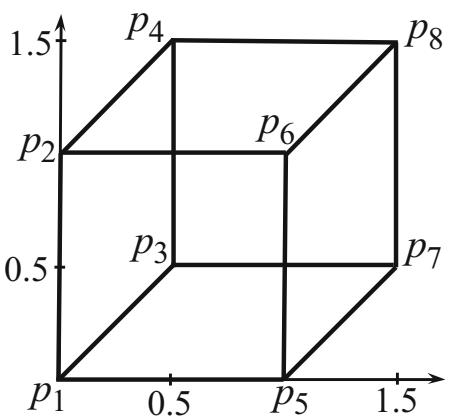

Fig. 8.

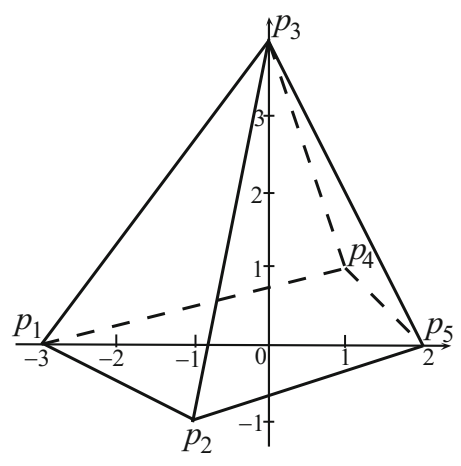

Fig. 7 .

Let us write the system of equations with respect to the vector $y=\left[y_{1}, y_{2}, \ldots, y_{8}\right]$ that follows from the absence of 'bends' of faces:

$$
\left.\begin{array}{l}
q_{1} \in q_{2} q_{5} q_{6} \Rightarrow y_{1}-y_{2}-y_{5}+y_{6}=0, \\
q_{5} \in q_{6} q_{7} q_{8} \Rightarrow y_{5}-y_{6}-y_{7}+y_{8}=0, \\
q_{3} \in q_{4} q_{7} q_{8} \Rightarrow y_{3}-y_{4}-y_{7}+y_{8}=0, \\
q_{1} \in q_{2} q_{3} q_{4} \Rightarrow y_{1}-y_{2}-y_{3}+y_{4}=0, \\
q_{1} \in q_{3} q_{5} q_{7} \Rightarrow y_{1}-y_{3}-y_{5}+y_{7}=0, \\
q_{2} \in q_{4} q_{7} q_{8} \Rightarrow y_{3}-y_{4}-y_{7}+y_{8}=0 .
\end{array}\right\}
$$

This system is equivalent to the following system, which expresses the variables $y_{4}, y_{6}, y_{7}, y_{8}$ in terms of $y_{1}, y_{2}, y_{3}, y_{5}$ :

$$
\left.\begin{array}{l}
y_{4}=y_{1}+y_{2}+y_{3}, \\
y_{6}=-y_{1}+y_{2}+y_{5}, \\
y_{7}=-y_{1}+y_{3}+y_{5}, \\
y_{8}=-2 y_{1}+y_{2}+y_{3}+y_{5} .
\end{array}\right\}
$$

Let us construct a system of inequalities that describes the visibility of the corresponding edges of the polyhedron. The edges $p_{1} p_{3}, p_{2} p_{6}, p_{3} p_{4}, p_{3} p_{7}, p_{5} p_{6}$, and $p_{6} p_{8}$ lie inside the polyhedron co $M_{P}$. Consequently, only the edges of the original polyhedron corresponding to them can be 'screened' by other faces and be invisible in some interpretations.

(1) The edge $q_{1} q_{3}$ can be 'screened' only by the face $q_{1} q_{2} q_{6} q_{5}$. Therefore, there is only one inequality that follows from the relation $p_{3}=0.25 p_{1}+0.5 p_{2}+0.25 p_{5}$ : 
(c1) $y_{3}<0.25 y_{1}+0.5 y_{2}+0.25 y_{5}=0$.

(2) The edge $q_{2} q_{6}$ can be 'screened' by the faces $q_{1} q_{2} q_{4} q_{3}$ and $q_{3} q_{4} q_{8} q_{7}$. In the first case, since the vertex $q_{2}$ is always visible, it is sufficient to write the inequality that follows from the impossibility of intersection of edges and faces. More exactly, the point $q_{6}$ must be 'in front of' the plane that extends the face $q_{1} q_{2} q_{4} q_{3}$. From the relation $p_{6}=-2 p_{1}-p_{2}+4 p_{3}$, we obtain

(c2) $y_{6}<-2 y_{1}-y_{2}+4 y_{3}$.

In the second case, when we consider the face $q_{3} q_{4} q_{8} q_{7}$, the relations $p_{2}=1.25 p_{4}+0.5 p_{7}-0.75 p_{8}$ and $p_{6}=0.25 p_{4}+0.5 p_{7}+0.25 p_{8}$ similarly yield two more inequalities:

$$
\begin{aligned}
& \text { (c3) } y_{2}<1.25 y_{4}+0.5 y_{7}-0.75 y_{8}, \\
& \text { (c4) } y_{6}<0.25 y_{4}+0.5 y_{7}+0.25 y_{8} .
\end{aligned}
$$

In what follows, we will briefly write only the edges, faces, and relations under consideration as well as the corresponding inequalities.

(3) The edge $q_{3} q_{4}$. The faces $q_{1} q_{2} q_{6} q_{5}$ and $q_{2} q_{4} q_{8} q_{6}$. The relations $p_{3}=0.25 p_{1}+0.5 p_{2}+0.25 p_{5}$, $p_{4}=-0.75 p_{1}+1.5 p_{2}+0.25 p_{5}$, and $p_{3}=1.5 p_{2}-p_{4}+0.5 p_{6}$. The inequalities

(c5) (c1),

(c6) $y_{4}<-0.75 y_{1}+1.5 y_{2}+0.25 y_{5}$,

(c7) $y_{3}<1.5 y_{2}-y_{4}+0.5 y_{6}$.

(4) The edge $q_{3} q_{7}$. The faces $q_{1} q_{2} q_{6} q_{5}$ and $q_{5} q_{6} q_{8} q_{7}$. The relations $p_{3}=0.25 p_{1}+0.5 p_{2}+0.25 p_{5}$, $p_{3}=2 p_{5}+2 p_{6}-3 p_{7}$, and $p_{7}=-0.75 p_{1}+0.5 p_{2}+1.25 p_{5}$. The inequalities

(c8) (c1),

(c9) $y_{3}<2 y_{5}+2 y_{6}-3 y_{7}$

(c10) $y_{7}<-0.75 y_{1}+0.5 y_{2}+1.25 y_{5}$.

(5) The edge $q_{5} q_{6}$. The faces $q_{1} q_{3} q_{7} q_{5}$ and $q_{3} q_{4} q_{8} q_{7}$. The relations $p_{5}=0.25 p_{4}+1.5 p_{7}-0.75 p_{8}$, $p_{6}=0.25 p_{4}+0.5 p_{7}+0.25 p_{8}$, and $p_{6}=-1.5 p_{1}+2 p_{3}+0.5 p_{5}$. The inequalities

(c11) $y_{5}<0.25 y_{4}+1.5 y_{7}-0.75 y_{8}$,

(c12) (c4),

(c13) $y_{6}<-1.5 y_{1}+2 y_{3}+0.5 y_{5}$.

(6) The edge $q_{6} q_{8}$. The faces $q_{3} q_{4} q_{8} q_{7}$. The relation $p_{6}=0.25 p_{4}+0.5 p_{7}+0.25 p_{8}$. The inequality (c14) (c4).

Thus, we have the following system of inequalities:

$$
\begin{aligned}
& \text { (c1) }-y_{1}-2 y_{2}+4 y_{3}-y_{5}<0 \text {, } \\
& \text { (c2) } 2 y_{1}+y_{2}-4 y_{3}+y_{6}<0 \text {, } \\
& \text { (c3) } 4 y_{2}-5 y_{4}-2 y_{7}+3 y_{8}<0 \text {, } \\
& \text { (c4) }-y_{4}+4 y_{6}-2 y_{7}-y_{8}<0 \text {, } \\
& \text { (c5) (c1), } \\
& \text { (c6) } 3 y_{1}-6 y_{2}+4 y_{4}-y_{5}<0 \text {, } \\
& \text { (c7) }-3 y_{2}+2 y_{3}+3 y_{4}-y_{6}<0 \text {, } \\
& \text { (c8) (c1), } \\
& \text { (c9) } y_{3}-2 y_{5}-2 y_{6}+3 y_{7}<0 \text {, } \\
& \text { (c10) } 3 y_{1}-2 y_{2}-5 y_{5}+4 y_{7}<0 \text {, } \\
& \text { (c11) }-y_{4}+4 y_{5}-6 y_{7}+3 y_{8}<0 \text {, } \\
& \text { (c12) (c4), } \\
& \text { (c13) } 3 y_{1}-4 y_{3}-y_{5}+2 y_{6}<0 \text {, } \\
& \text { (c14) (c4). }
\end{aligned}
$$




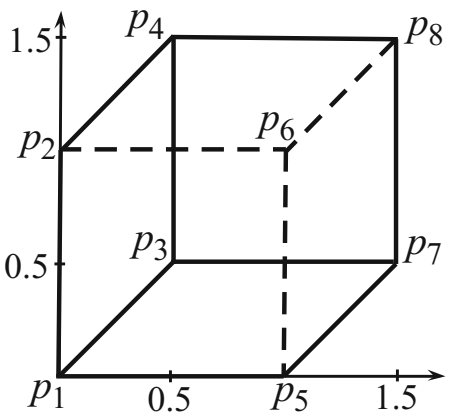

Fig. 9.

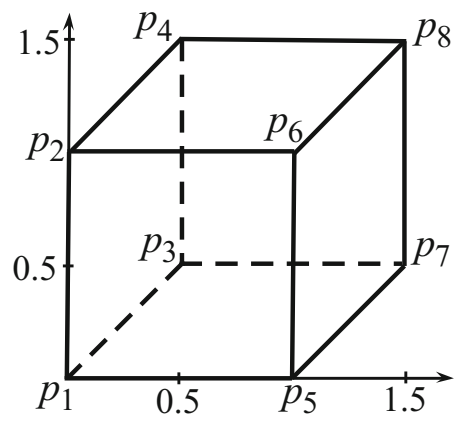

Fig. 10.

In view of the above equalities that express the unknowns $y_{4}, y_{6}, y_{7}, y_{8}$ in terms of the remaining variables, this systems turns into the following system (here, $h(y)=-y_{1}-2 y_{2}+4 y_{3}-y_{5}$ ):

$$
\begin{aligned}
& \text { (c1) } \quad h(y)<0 \text {, } \\
& \text { (c2) }-h(y)<0 \text {, } \\
& \text { (c3) }-h(y)<0 \text {, } \\
& \text { (c4) }-h(y)<0 \text {, } \\
& \text { (c6) } \quad h(y)<0 \text {, } \\
& \text { (c7) } \quad h(y)<0 \text {, } \\
& \text { (c9) } \quad h(y)<0 \text {, } \\
& \text { (c10) } \quad h(y)<0 \text {, } \\
& \text { (c11) }-h(y)<0 \text {, } \\
& \text { (c13) }-h(y)<0 \text {. }
\end{aligned}
$$

This system has only two MFSs: (c1), (c6), (c7), (c9), (c10) and (c2), (c3), (c4), (c11), (c13). Let us list inequalities whose validity is necessary for the visibility of the corresponding edges: $(\mathrm{c} 1)$ for the edge $q_{1} q_{3} ;(\mathrm{c} 1)$, (c9), and (c10) for the edge $q_{3} q_{7} ;(\mathrm{c} 2),(\mathrm{c} 3)$, and (c4) for the edge $q_{2} q_{6} ;(\mathrm{c} 4),(\mathrm{c} 11)$, and (c13) for the edge $q_{5} q_{6} ;(\mathrm{c} 1)$, (c6), and (c7) for the edge $q_{3} q_{4}$; and (c4) for the edge $q_{6} q_{8}$. Thus, the first MFS corresponds to the interpretation in which the edges $q_{1} q_{3}, q_{3} q_{4}$, and $q_{3} q_{7}$ are visible (Fig. 9), whereas the second MFS corresponds to the interpretation in which the edges $q_{2} q_{6}, q_{5} q_{6}$, and $q_{6} q_{8}$ are visible (Fig. 10).

Thus, the visible faces in the first interpretation are $q_{1} q_{2} q_{4} q_{3}, q_{3} q_{4} q_{8} q_{7}$, and $q_{1} q_{3} q_{7} q_{5}$, and the visible faces in the second interpretation are $q_{1} q_{2} q_{6} q_{5}, q_{5} q_{6} q_{8} q_{7}$, and $q_{2} q_{4} q_{8} q_{6}$.

\section{CONCLUSIONS}

In our opinion, the proposed approach to the recovery of images holds much promise but needs further theoretical investigation. Its advantages are, first of all, the computational simplicity (for two interpretations, it is sufficient to calculate only one coefficient of inequalities) and the possibility of extension to more general problems of image interpretation. Note that the approach is not restricted to convex polyhedra; this case was taken for simplicity.

However, we should note that the technique considered in this paper does not take into account the metric properties of a three-dimensional object, which are important for practical problems. Therefore, it will be necessary to further develop the proposed approach in combination with the classical projective approach. 


\section{ACKNOWLEDGMENTS}

This work was supported by the Russian Foundation for Basic Research (project no. 10-0100273) and by the Presidium of the Ural Branch of the Russian Academy of Sciences (project nos. 12-P-1-1016, 12-S-1-1017/1).

\section{REFERENCES}

1. V. N. Kozlov, "Recognition of images represented by a finite set of points," J. Math. Sci. (N. Y.) 172 (5), 690-699 (2011).

2. D. V. Yurin, "Modern concept of recovering three-dimensional scenes from a collection of digital images: Filling virtual reality systems," in Three-Dimensional Vizualization of Scientific, Technical, and Social Reality: Cluster Modeling Technologies (Udmurtsk. Gos. Univ., Izhevsk, 2009), Vol. 1, pp. 96-100 [in Russian].

3. R. Hartley and A. Zisserman, Multiple View Geometry in Computer Vision (Cambridge Univ. Press, Cambridge, 2004).

4. Make3D Publications, http://make3d.cs.cornell.edu. Cited June 30, 2012.

5. I. A. Evin, Brain Synergetics (Regulyarn. Khaotichesk. Dinamika, Izhevsk, 2005) [in Russian].

6. R. L. Gregori, The Intelligent Eye (Weidenfeld \& Nicolson, London, 1970; Mir, Moscow, 1972).

7. G. Caglioti, From Perception to Thought (Mir, Moscow, 1998) [in Russian].

8. L. Shapiro and G. Stokman, Computer Vision (Prentice Hall, Upper Saddle River, NJ, 2001; Binom, Moscow, 2006).

9. I. I. Eremin, Systems of Linear Inequalities and Linear Optimization (Ural'sk. Otdel. Ross. Akad. Nauk, Yekaterinburg, 2007) [in Russian]

10. I. I. Eremin, V. D. Mazurov, and N. N. Astaf'ev, Improper Problems of Linear and Convex Programming (Nauka, Moscow, 1983) [in Russian].

11. V. D. Mazurov, Mathematical Methods of Pattern Recognition (Izd. Ural'sk. Gos. Univ., Sverdlovsk, 1982) [in Russian].

12. Vl. D. Mazurov, M. Yu. Khachai, and V. S. Sharf, "On balance and imbalance," in Mathematical Methods of Pattern Recognition: Proc. All-Russia Conf. (MAKS, Moscow, 2009), pp. 70-73 [in Russian].

13. Vl. D. Mazurov, "Collective behavior and mathematical psychology," in Economic Development in the Modern World (Izd. Ural'sk. Gos. Univ., Ekaterinburg, 2009), pp. 112-116 [in Russian].

14. V. D. Mazurov and A. I. Smirnov, "On the algebraical approach to the recovery of objects from their images," in Automated Systems for Image Processing: Abstracts All-Union Conf. (Nauka, Moscow, 1986), p. 154 [in Russian].

15. V. D. Mazurov and A. I. Smirnov, "Contradictory interpretation of ambiguous scenes," in Mathematical Programming Methods and Their Computer Implementation (Ural'sk. Nauchn. Tsentr Akad. Nauk SSSR, Sverdlovsk, 1984), pp. 72-73 [in Russian].

Translated by E. Vasil'eva 